\title{
Evaluative Conditioning: Recent Developments and Future Directions
}

\author{
Anne Gast ${ }^{1}$, Bertram Gawronski ${ }^{2}$, and Jan De Houwer ${ }^{1}$ \\ ${ }^{1}$ Ghent University, Belgium \\ ${ }^{2}$ The University of Western Ontario, Canada
}

Author Note

Anne Gast, Department of Experimental Clinical and Health Psychology, Ghent University, Belgium; Bertram Gawronski, Department of Psychology, The University of Western Ontario, Canada; Jan De Houwer, Department of Experimental Clinical and Health Psychology, Ghent University, Belgium.

Preparation of this article was supported by Methusalem Grant BOF09/01M00209 from Ghent University to Jan De Houwer and by a VBO Travel Grant from Ghent University to Jan De Houwer and Bertram Gawronski. The special interest meeting that led to the current special issue was funded by the Research Foundation - Flanders (FWO) by means of the research community WO.001.07N "Automatic processes in psychopathology and health related behavior". We thank Agnes Moors for valuable discussions.

Correspondence concerning this article should be addressed to Anne Gast, Department of Experimental Clinical and Health Psychology, Faculty of Psychology and Educational Sciences, Ghent University, Henri Dunantlaan 2, B-9000 Ghent, Belgium, Email: Anne.Gast@UGent.be, Phone: +32 (9) 264-6444. 


\begin{abstract}
Evaluative conditioning (EC) is generally considered to be one of the routes via which likes and dislikes are acquired. We identify recent trends in EC research and speculate about the topics that will dominate future research on EC. Many of the recent developments in EC research were shaped by functional definitions of EC that refer only to environmental conditions, but not to mental processes and representations. These functional definitions stimulated the development of and debates between theories about the mental processes that mediate EC. These trends, as well as greater methodological and conceptual sophistication, inspired meta-conditional studies that aim at determining the conditions under which EC is characterized by certain properties. The question of whether EC depends on contingency awareness remains a central topic, albeit with a stronger link to theorizing about underlying mental processes. Other automaticity features have gained enhanced interest, as can for example be seen in research investigating whether EC is goal-independent and whether EC is resource-independent. We argue that future research would benefit from a sharper distinction between processes that operate during acquisition (i.e., when CS-US pairs are presented) and processes that operate during measurement (i.e., when the effects of CS-US pairings are assessed). We conclude with a short summary of all articles in this Special Issue, relating them to the identified trends in EC research.
\end{abstract}

Keywords: automaticity, evaluative conditioning, functional definition, mental process theories 
Many psychologists have devoted their careers to understanding the likes and dislikes that humans (and non-human animals) display. Understanding these evaluations is important not only for its own sake, but also because they are assumed to determine various kinds of behavior, ranging from the purchase of consumer products to the pursuit of potential mates. Learning more about how evaluations are acquired and how they can be changed could thus help us understand and control many aspects of human behavior. For almost four decades, evaluative conditioning (EC) researchers have examined one potential way of creating and changing likes and dislikes: the pairing of stimuli (cf. Levey \& Martin, 1975). More specifically, research has shown that the liking of a stimulus can be increased by pairing it with positive stimuli and decreased by pairing it with negative stimuli (for a meta-analysis, see Hofmann, De Houwer, Perugini, Baeyens, \& Crombez, 2010).

The aim of this article, and the Special Issue that it introduces, is to provide an overview of recent trends in EC research and to speculate about potential future developments. The need for such an update became clear to us during a recent Special Interest Meeting on EC that took place at Ghent University (Belgium) in December 2010. Whereas earlier meetings on EC focused to a large extent on whether genuine EC effects can be observed and on the boundary conditions of these effects (see De Houwer, Baeyens, \& Field, 2005), the most recent meeting at Ghent University was dominated by lively debates about the exact nature of EC and the mental processes and representations that underlie the effect. Recurring questions, such as the role of conscious awareness in EC, are now addressed in a manner that is both conceptually and methodologically more sophisticated than ever. In the following section, we provide an overview of these 
developments and current issues of debate. Expanding on this overview, we then offer some projections on how these trends could influence future research on EC. In the final section, we briefly review the articles in this Special Issue, elucidating how they relate to the identified trends and how they forebode future hot topics in EC research.

\section{Recent developments in EC research}

\section{Defining EC}

Until recently, research on EC has been dominated by a strong empirical focus with little consideration of basic conceptual issues. Although early studies offered many valuable insights (see De Houwer, Thomas, \& Baeyens, 2001, for a review), the communication of these findings has been characterized by inconsistent use of terminology. As De Houwer (2007) pointed out, different researchers conceived of EC in different ways, which can hamper theorizing and the interpretation of empirical findings. Whereas some researchers considered EC as the effect of stimulus pairings on liking, others conceptualized it as a mental mechanism that produces changes in liking by creating associations in memory. To avoid conflations between behavioral effects and mental constructs that mediate these effects, De Houwer argued that EC is best defined as an effect, that is, as "a change in the valence of a stimulus that results from pairing the stimulus with another stimulus" (p. 230). De Houwer's definition and similar conceptualizations (e.g., Jones, Olson, \& Fazio, 2010) are now widely used and have greatly facilitated the interpretation of empirical findings and theoretical debates about the mechanisms underlying EC. ${ }^{1}$ 
The proposed conceptualizations of EC as an effect can be described as functional definitions, in that they define $\mathrm{EC}$ in terms of elements in the environment (stimulus pairings) without referring to mental processes and representations (e.g., formation of associative links; acquisition of propositional knowledge). The lack of reference to mental constructs has two important advantages (see De Houwer, 2007, 2011a). First, it is easier to verify whether stimulus pairings produce changes in liking than to verify whether certain mental constructs mediate the impact of stimulus pairings on liking. Second, functional definitions of EC do not restrict on an a priori basis ideas about the mental processes and representations that might mediate the effect of stimulus pairings on liking. According to functional definitions, EC as a basic phenomenon does not depend on whether it is explained by the formation of associative links (e.g., Gawronski \& Bodenhausen, 2006), the acquisition of propositional knowledge (e.g., De Houwer, 2009), or any other mental mechanism (for a review, see De Houwer, 2011b). The question of which mental processes and representations mediate EC effects, should be distinguished from the question of what EC is as a basic phenomenon. Hypotheses about underlying processes and representations can be answered only by testing theoretically derived predictions about the moderators of EC.

Despite these advantages, a number of questions can be raised about the functional definitions of EC that are currently available. For example, the definition offered by De Houwer (2007) states that changes in valence qualify as EC effects if they can be attributed to stimulus pairings. However, neither the term pairings nor the term valence change are as unambiguous as it might seem. This ambiguity has sparked several 
debates at the 2010 meeting in Ghent. In this section, we provide a brief discussion of the issues that have been raised.

Do effects due to pairings include priming?

A first ambiguity that was discussed during the meeting in Ghent is that the above definition might be understood to include priming effects. In a typical affective priming procedure, participants' task is to give an evaluative response to a target stimulus that is presented shortly after a prime stimulus (e.g., Fazio, Sanbonmatsu, Powell, \& Kardes, 1986; Payne, Cheng, Govorun, \& Stewart, 2005). It can thus be argued that prime and target are presented in pairs. Because a valenced prime typically influences participants' evaluations of the target (e.g., how quickly or how positively they evaluate the target), De Houwer's (2007) definition of EC can be interpreted as subsuming priming effects: prime and target stimuli are paired and this pairing influences the evaluation of the target. Considering, however, that EC is a form of learning, it becomes clear that EC, like all instances of learning, refers to the impact of past events on current behavior. Hence, any definition of EC should be limited to changes in liking that are due to the prior pairing of stimuli. Because in priming tasks, the change in liking is observed during the pairing of the prime and target (i.e., at the time of the first occurrence of the target after the prime), there is no past pairing that can lead to the change in liking. Thus, there are reasons to argue that De Houwer's (2007) definition does not include priming effects. Nevertheless, one could resolve any ambiguity on this matter by modifying his definition in the following manner: "EC is a change in the valence of a stimulus that results from a previous pairing of the stimulus with another stimulus." Note that a similar 
clarification would have to be applied to functional definitions of other types of (classical and operant) conditioning. For example, fear conditioning also refers to effects of prior CS-US pairings and therefore has to be tested (e.g., by assessing skin conductance) in the absence of the US (e.g., shock).

What about the effects of instructions?

Another issue that sparked discussions is whether changes in valence based on instructions about pairings should be considered as instances of EC. There are several possible reasons for excluding effects of instructions from the definition of EC. First, it is obvious that instructions about pairings are different from actual CS-US pairings, in that only the latter involve the physical presence of the CS and the US. A typical instruction only involves a statement about the co-occurrence of two stimuli (e.g., the instruction that the nonword 'enanwal' will be followed by a positive picture; see De Houwer, 2006). Nevertheless, it has been argued that the words appearing in instructions about pairings can be argued to be CSs and USs themselves (Field, 2006; Mineka \& Zinbarg, 2006). Hence, although the number of pairings in such instructions is typically reduced to one, instructions can be said to contain a single CS-US pairing.

Another argument for rejecting effects of instructions as instances of EC is the fact that instructions typically involve more than a simple pairing of stimuli. In many cases, they also contain information about the way stimuli are related (e.g., CS is paired with US, CS has the same meaning as US). There are reasons to believe that this relational information determines the effects of instructions. For example, the sentences "CS1 will be paired with a positive US" and "CS1 will not be paired with a positive US" 
both involve the physical pairings of the verbal stimuli "CS1" and "positive US", but may have different effects on liking (cf. Deutsch, Gawronski, \& Strack, 2006; Gawronski, Deutsch, Mbirkou, Seibt, \& Strack, 2008). One could, however, argue that many, if not all instances of EC involve relational information. It is important to realize that relational information about a CS-US pair can be provided not only by words (e.g., “is paired with") but also by other stimuli whose learning history or current properties suggest a specific type of stimulus relation (e.g., Zanon, De Houwer, \& Gast, 2012, this issue). Even the mere fact that stimuli appear together on a screen could be a cue that the stimuli are related in a certain way (e.g., that they belong together; Hayes, BarnesHolmes, \& Roche, 2001). Hence, linking the definition of EC to the absence of relational information would render it difficult to verify the definition because it is difficult to determine that relational information is actually absent in a certain situation. We therefore prefer to think of cues about the relation between stimuli (e.g., words, symbols, context) as potential moderators of the effect of stimulus pairings on liking (e.g., Fiedler \& Unkelbach, 2011; Förderer \& Unkelbach, 2011; Zanon et al., 2012, this issue). Rather than excluding relational information from research on EC, we believe that our understanding of the impact of stimulus pairings on liking can be enriched by studying how cues about the type of stimulus relation moderate this impact.

A third argument for rejecting instruction effects as instances of EC is that these effects might be mediated by propositional processes whereas "genuine" EC effects must be due to the automatic formation of associations. A major problem with this argument is that it requires an exclusion of all procedures in which EC effects are driven by propositional processes. However, this decision cannot be made merely by inspecting the 
procedure or the behavior that is elicited by the procedure (De Houwer, 2011a). In our opinion, the contribution of associative and propositional processes to EC is the subject of empirical research and theoretical debate about the mechanisms underlying EC. Hence, their presence versus absence should not be used as the criterion for classifying changes in liking as instances of EC. As we indicated above, defining EC in terms of mental processes and representations would not only render it difficult to verify whether a change in liking is an instance of EC, but also hinder progress in our understanding of the mental processes and representations that mediate EC effects. ${ }^{2}$

A final reason for not accepting instruction effects as instances of EC is that it would blur the distinction between EC and other phenomena such as persuasion. Persuasion can be defined as a change in liking as the result of verbally communicated messages (for a review, see Johnson, Maio, Smith-McLallen, 2005). EC via instructions would count as an instance of persuasion because it involves changes in liking as the result of a verbal message. A distinction between the two phenomena in terms of the presence or absence of verbal instructions seems relatively straightforward. For example, one could limit EC to cases that involve the actual presentation of stimuli pairings in a format other than sentences and reserve the term persuasion for all cases that involve the presentation of evaluative information in the form of sentences. However, questions could be raised about whether sentences differ in a fundamental manner from situations in which stimuli are presented in the presence of nonverbal cues about the relation between the stimuli (see Hayes et al., 2001). An alternative option is to accept a certain degree of overlap between instances of EC and instances of persuasion. Such an approach 
could foster interactions between research on both phenomena, highlighting that they might (sometimes) be mediated by similar mental processes.

Regardless of whether one prefers to include or exclude instruction effects in the conceptual definition of EC, we argue that any demarcation should be made in functional terms, that is, by means of elements in the environment rather than the mental constructs that are assumed to underlie EC effects. Moreover, we believe that it is useful to compare the effects of instructions with the effects of "actual" pairings irrespective of one's conceptual preference. To the extent that both display the same functional properties, there is reason to believe that they are due to the same underlying mechanisms (e.g., acquisition of propositional knowledge). However, if the two effects are characterized by different functional properties, any mental process account (e.g., single-process associative, single-process propositional, or dual-process account) would have to explain why instructions and "actual" pairings have different effects. Because ignoring the effects of instructions on liking would limit the empirical basis for theorizing about the mechanisms underlying EC, we think that it is useful to compare the functional properties of instruction effects with the ones of "actual" pairing effects.

\section{Does a functional definition include demand effects?}

Some researchers might be worried that a functional definition that does not impose any restrictions on the processes by which pairings lead to a change in liking might not be able to distinguish genuine EC from demand effects. There are several possibilities for dealing with demand effects at the conceptual level. First, one could argue that demand effects do not involve a genuine change in liking, and thus do not 
qualify as instances of EC. However, such a proposal begs the question of what constitutes a "genuine" change in liking. Although the change in liking is the one feature that distinguishes EC from other instances of classical conditioning (see De Houwer, 2007), this question has received little attention in the literature on EC. Hence, any definition of EC has to ultimately deal with the question of what constitutes a "genuine" change in liking.

Second, one could argue that changes in liking due to demand effects are genuine instances of EC (i.e., effects of stimulus pairings on liking), but that such instances of EC are due to a special type of mediating mental process (i.e., participants intentionally behave in a way that meets the perceived expectations of the experimenter). Empirically, one can exclude the operation of these processes (and thus demand effects) by removing the opportunity for these processes to operate. Typically, this is done by using measures of liking that are difficult to control. If EC is still found under those conditions, it is likely not due to demand. Note, however, that it is not possible to draw the opposite conclusion if EC effects emerge on measures that are relatively easy to control, but not on measures that are relatively difficult to control. After all, a null effect on the latter type of measure could be due to multiple factors, including low reliability of the measure (for a detailed discussion, see Gawronski \& De Houwer, in press). Thus, claims about demand can be interpreted as hypotheses about mental processes, and these hypotheses imply specific predictions about the convergence versus divergence of measurement outcomes. To the extent that these predictions are disconfirmed, demand can be ruled out as a potential mechanism. However, demand cannot be confirmed in a positive manner, just as it is impossible to verify the truth of any theoretical hypothesis (Popper, 1934). From this 
perspective, the concept of demand does not involve any problems for a functional definition of EC, because it is not an empirical effect that needs to be distinguished from "genuine" EC. Instead, it is a theoretical hypothesis about mental mechanisms that needs to be ruled out by investigating the boundary conditions of the observed effects (e.g., by comparing EC effects across different measures).

\section{Theoretic developments}

Functional definitions of EC highlight the fact that EC can in principle be due to any type or combination of mental processes. This insight was undoubtedly one of the factors that spurred recent advances in theorizing about the mental processes and representations that mediate EC. During the past years, the theoretical landscape of EC has been enriched by a number of new accounts (for reviews, see De Houwer, 2011b; Jones, Olson, \& Fazio, 2010). One of them is the implicit misattribution account (Jones, Fazio, \& Olson, 2009), which assumes that the evaluative response that is elicited by the US and experienced during CS-US pairings is implicitly misattributed to the CSs. This misattribution is claimed to produce a stimulus-response (S-R) link between the representation of the CS and the evaluative response. Another explanation that has been influential during the last years is the propositional account of EC (De Houwer, 2009; Mitchell, De Houwer, \& Lovibond, 2009). This account posits that changes in the evaluation of the CS are due to the acquisition and validation of propositional knowledge about the relation between the CS and the US. Unlike associations, propositions are qualified links that are represented in the form of statements about events in the world.

Last, but not least, dual-process models of information processing postulate that EC can 
be the result of either associative or propositional processes, with two processes being characterized by specific patterns of mutual interactions (Gawronski \& Bodenhausen, 2011).

The idea that associative and propositional processes interact in producing EC effects fits well with a meta-conditional approach to EC. Rather than trying to identify general properties of EC (e.g., whether or not EC depends on contingency awareness), the meta-conditional approach aims to determine the conditions under which EC has certain properties (De Houwer, 2007). This implies that different instances of EC might have different functional properties (e.g., De Houwer, 2007; Gawronski \& Bodenhausen, 2011; Jones et al., 2010). For example, instances of EC that are due to the formation of associative links might be independent of awareness of the CS-US contingencies, whereas instances of EC that are produced by propositional processes might depend on contingency awareness (Gawronski \& Bodenhausen, in press). For example, Gawronski and LeBel (2008) found EC effects on both an explicit measure (i.e., evaluative ratings) and an implicit measure (i.e., evaluative priming) when participants were instructed to adopt an affective focus. However, when participants were instructed to adopt a cognitive focus, EC effects emerged only on the implicit, but not on the explicit measure. Similarly, recent research has shown that US-revaluation influences liking of a CS only when the CS was paired with a single US, but not when it was paired with multiple USs (Sweldens, Van Osselaer, \& Janiszewski, 2010). These results suggest that EC is sometimes based on stimulus-stimulus learning and sometimes on stimulus-response learning (see also Gast \& Rothermund, 2011a; Walther, Gawronski, Blank, \& Langer, 2009). The important task 
for future theorizing is to explain why EC effects are moderated in the observed manner and to generate novel predictions that could be used to test the proposed explanations.

\section{Increased methodological and conceptual sophistication}

Another important development in EC research is that newly established methods allow researchers to be much more precise in their conclusions from a given set of data. This is particularly true for research on contingency awareness where several authors have made important contributions to enhanced methodological and conceptual precision.

Until recently, the relation between conscious knowledge of stimulus pairings and EC effects has typically been assessed at the level of the participant, for example by comparing EC effects of participants who remember more than half of the presented CSUS pairings with those of participants who remember less than half (e.g. Fulcher \& Hammerl, 2001). Pleyers, Corneille, Luminet, and Yzerbzt (2007) pointed out that it is more precise to consider knowledge about the pairings at the level of a given stimulus pair. As a result, analyses at the level of stimulus pairs have largely replaced analyses at the level of the participant as the dominant analytical tool.

Another important distinction is whether a participant is aware of the identity of the US that had been paired with a CS (identity awareness) or whether the participant remembers only the valence of the relevant US (valence awareness). Stahl, Unkelbach, and Corneille (2009) argued that valence awareness is more important than identity awareness in determining EC. However, some researchers raised concerns that measures of valence memory might not always provide a valid indication of conscious awareness of CS-US pairings. Specifically, a correct response on a measure of valence memory 
might indicate actual knowledge of the CS-US pairing, but it might also reflect valencebased guessing (Bar-Anan, De Houwer, \& Nosek, 2010; Gawronski \& Walther, 2012; Hütter, Sweldens, Stahl, Unkelbach, \& Klauer, in press). That is, participants may use their conditioned (dis)liking of the CS as a cue for guessing the valence of the US it had been paired with. Such a strategy could artificially increase the strength of the relation between valence memory and EC. A recent study by Hütter et al. (in press) has made an important step toward resolving this problem by using multinomial modeling (Batchelder \& Riefer, 1999) to disentangle the role of actual CS-US memory and valence-based guessing in EC.

Another important concern is that conscious knowledge during the acquisition phase (i.e., when CS-US pairings are presented) needs to be distinguished from conscious knowledge during the measurement phase (i.e., when the effects of CS-US pairings are tested). Although this problem has been identified repeatedly in research on EC (Baeyens, Eelen, \& Van den Bergh, 1990; Purkis \& Lipp, 2001; Shanks \& St. John, 1994), it is still common practice to investigate contingency awareness with measures of recollective memory at the end of the experiment. Recently, this problem has received more attention, because different theoretical accounts make different predictions about the role of contingency knowledge during acquisition versus measurement (Bar-Anan et al., 2010; Gast, De Houwer, \& De Schryver, 2012, this issue; Gawronski \& Walther, 2012). Although measures of recollective memory are well suited to assess participants' conscious knowledge at the time of measurement, they are not suited to address the old question of whether EC effects require conscious awareness of CS-US pairings during their presentation. As outlined by Gawronski and Walther (2012), the latter question 
requires alternative approaches in which awareness of CS-US pairings is manipulated experimentally. Ideally, such studies would also include online measures of conscious awareness during the presentation of CS-US pairings (e.g., Baeyens et al., 1990; Purkis \& Lipp, 2001) to resolve the identified ambiguities of recollective memory measures.

\section{Automaticity features: Awareness and beyond}

The preceding section shows that the role of contingency awareness remains a central topic in EC research. Yet, different from earlier studies on this question, contingency awareness is not studied for its own sake (i.e., to determine whether EC can occur unconsciously), but to gain information about the automatic (or non-automatic) nature of the processes underlying EC effects. Automaticity is often seen as an important dimension that distinguishes between different mental process models of EC, in particular associative versus propositional models (e.g., De Houwer, 2009; Gawronski \& Bodenhausen, 2006). Recent approaches, however, do not conceptualize automaticity as a unitary concept. Rather, automaticity is an umbrella concept that encompasses multiple different features (e.g., Moors, \& De Houwer, 2006). Whether participants have to be consciously aware of the stimulus pairings is therefore not the only relevant question regarding the automaticity of EC effects and their underlying mental processes. Thus, in line with more fine-grained conceptualizations, EC researchers have started examining automaticity features other than awareness, including the impact of goals (e.g., Corneille, Yzerbyt, Pleyers, \& Mussweiler, 2009; Gast \& Rothermund, 2011b; Verwijmeren, Karremans, Stroebe, \& Wigboldus, 2012, this issue), attention (e.g., Blask, Walther, Halbeisen, \& Weil, 2012, this issue; Field \& Moore, 2005; Fulcher \& Hammerl, 2001; 
Kattner, 2012), and cognitive resources (e.g., Dedonder, Corneille, Yzerbyt, \& Kuppens, 2010; Pleyers, Corneille, Yzerbyt, \& Luminet, 2009; Walther, 2002).

\section{Possible future trends: Automaticity during acquisition and measurement}

Speculating about future trends is always a risky business. Yet, a relatively riskfree prediction is that the trends that we have identified in recent research will continue to dominate research for the coming years. Although the adoption of functional definitions has led to major progress in ideas about the nature of EC and theorizing about its underlying mechanisms, the debate about the mental processes and representations that mediate EC is far from settled. Recent methodological advances and increased conceptual precision will likely be a great asset in testing the predictions of competing accounts. Because neuroscientific methods are gaining importance in virtually all areas of psychology, it also seems safe to assume that these methods will play a greater role in research on evaluative conditioning.

There is, however, one prediction for which we want to go out on a limb. Although the idea that one should make a distinction between processes that operate during acquisition (i.e., when CS-US pairings are presented) and processes that operate during measurement (i.e., when the effects of CS-US pairings are tested) is certainly not novel, it is only recently that the implications of this idea have received more attention (e.g., Gast et al., this issue; Gawronski \& Walther, 2012). Whereas previous research applied this distinction only to the role of contingency awareness in EC, we believe that it can provide important insights also into the influence of other features of automaticity. A clear methodological and theoretical distinction between the factors and processes that 
have an influence during the presentation of CS-US pairings and those that have an influence during the measurement of CS evaluations could help to resolve some ongoing debates about the mental processes and representations underlying EC effects. If we accept the distinction between processes that operate during acquisition and those that operate during measurement, we should also accept the idea that both types of processes can possess different features of automaticity. In the following sections, we briefly discuss this issue with regard to two automaticity features: the goal-independence and resource-independence of EC effects.

\section{Goal independence}

When considering the impact of goals on processes or effects, a distinction can be made between proximal and distal goals. In the context of EC, proximal goals are those that relate to the impact of stimulus pairings on changes in liking, and thus directly to the EC effect. In contrast, distal goals can be conceptualized as goals that do not directly relate to the impact of stimulus pairings on liking, but may nevertheless influence EC effects. If EC effects are unaffected by proximal or distal goals, they can be described as goal-independent, and thus as automatic in this particular sense. Yet, EC effects would be goal-dependent, and thus non-automatic in this particular sense, if they are influenced by proximal or distal goals.

There are several proximal goals that may be relevant for EC, including goals to produce or promote an effect or process and goals to stop or prevent an effect or process. If a proximal goal has an impact on EC (e.g., when the goal not to show an impact of stimulus pairings on liking eliminates or reduces EC), EC can be described as controlled 
(Moors, Spruyt, \& De Houwer, 2010). However, when considering the distinction between processes that operate during acquisition and those that operate during measurement, it becomes clear that the concept of control can refer to either of these processes. For example, a preventive goal during the presentation of CS-US pairings could involve the goal not to be influenced by the pairings. In contrast, a preventive goal during the measurement of CS evaluations could be the goal to conceal the likes or dislikes that were acquired during the learning phase. Similarly, a promotive goal during the presentation of CS-US pairings could be the goal to learn the pairings well or to pay attention to all information relevant for the evaluation of the CSs. In contrast, a promotive goal during the measurement of CS evaluations could be to express or exaggerate likes and dislikes that are (or could have been) due to CS-US pairings. Balas and Gawronski (2012, this issue) provided evidence that the impact of proximal goals to promote versus prevent the influence of CS-US pairings at the time of measurement is moderated by contingency memory. In their research, instructions to maximize or minimize the influence of CS-US pairings moderated EC effects in line with task instructions. However, this influence was observed only when participants were able to recall the valence of the US that had been paired with a given CS. When participants failed to remember the valence of the US, significant EC effects emerged regardless of control instructions.

Different from proximal goals, distal goals in EC can be described as goals that not directed at the impact of stimulus pairings on liking. A useful example of a distal goal can be found in the research by Verwijmeren et al. (2012, this issue). The authors showed that thirst (i.e., the goal to drink) moderates the relative strength of EC effects based on 
goal relevant stimulus relations (beverage-disgusted face) as compared to goal irrelevant stimulus relations (beverage-fearful face). Similar to the proposed distinction for proximal goals, it is possible to consider the influence of a distal goal during the acquisition phase and the measurement phase. In the Verwijmeren et al. study, for example, participants' goal-state (i.e., their degree of thirst) was equivalent during the presentation of the CS-US pairings and during the measurement of CS evaluations. Hence, it is possible that the goal-state influenced EC effects through processes that operate during the presentation of CS-US pairings. However, it is also possible that thirst influenced EC effects during the measurement of CS evaluations (e.g., Ferguson \& Bargh, 2004; Seibt, Häfner, \& Deutsch, 2007).

\section{Resource independence}

Another important feature of automaticity is resource-independence. A process or effect can be called resource-independent, or efficient, if it requires little or no attentional capacity (Moors \& De Houwer, 2006; Moors et al., 2010). The resource-independence of a process or effect is typically demonstrated by showing that it is not disrupted through simultaneous performance of a secondary task. In contrast, a process or effect can be described as resource-dependent, and thus non-automatic in this particular sense, if it is disrupted by simultaneous cognitive activities. As with the operation of goals, a distinction should be made between the resource-independence of processes that operate during the acquisition phase and those that operate during the measurement phase.

Research on the influence of secondary tasks during the presentation of CS-US pairings is relevant for the resource-independence of the processes that operate during the 
acquisition phase (e.g., Dedonder et al., 2010; Pleyers et al., 2009; Walther, 2002). It has to be noted, however, that potential effects of a secondary task could also be due to differences in task-related goals. For example, comparing conditions in which participants are asked to rehearse an 8-digit string to conditions in which they are not asked to rehearse a digit string involves a confound between task-related goals and cognitive resources. A cleaner way of investigating the role of cognitive resources is to keep participants' goals constant across conditions, and to manipulate only the demands of the secondary task (e.g., by asking participants to rehearse an 8-digit string vs. a 2digit string). Furthermore, manipulating cognitive resources by means of attentional tasks might also influence the acquisition of conscious knowledge about the pairings. In such cases, the effect of the secondary task manipulation might be indirect in the sense that it is mediated by conscious knowledge about the pairings (Dedonder et al., 2010; Pleyers et al., 2009). These ambiguities require sophisticated methods to disentangle the effects of attention, goals, resources, and knowledge. An interesting example in this regard is a study by Blask et al. (2012, this issue) who manipulated attention in a bottom-up manner that is less likely to influence goals.

Expanding on the question of whether EC effects are moderated by the availability of cognitive resources during the acquisition phase, another interesting question concerns the resource-independence of the processes during the measurement phase. A possible approach in this regard might be to compare results on implicit and explicit measures. Potential dissociations between the two kinds of measures, however, have to be interpreted with caution, because several other factors, and in particular the presence or absence of other automaticity features, might differentially influence 
responses on the two kinds of measures (Moors et al., 2010). For example, whereas selfpresentation goals may influence EC effects on evaluative ratings, such goals are less likely to influence EC effects on evaluative priming tasks. Thus, a cleaner way to investigate the role of cognitive resources during the measurement phase would be to use identical measures under conditions of high versus low distraction by a secondary task (e.g., evaluative ratings while rehearsing an 8-digit string vs. a 2-digit string).

\section{This issue}

Many of the identified trends are reflected in the contributions that are featured in this Special Issue. In the final section of this article, we briefly summarize these contributions and relate them to the themes discussed above.

Balas \& Gawronski (2012, this issue) instructed participants to either maximize or minimize EC effects on valence ratings. The authors showed that this instruction influenced EC effects in line with task instructions. However, a moderating effect of task instructions was observed only for those CS-US pairs that were remembered by participants. These results suggest that participants can intentionally control EC effects on evaluative ratings. However, as pointed out by the authors, the set-up of their study allowed them to draw conclusions about the influence of intentional control during the measurement of conditioned CS evaluations, but not about the role of intentional control during the conditioning phase. Another important finding is that, while participants' ability to control EC effects depended on their memory of the CS-US pairings, the EC effect itself was independent of memory. In line with dual-process accounts of EC, this result may be interpreted as evidence for the operation of two distinct processes: one 
process that depends on conscious knowledge of CS-US pairings and another one that is independent of such knowledge.

Blask, Walther, Halbeisen, and Weil (2012, this issue) manipulated two attentionrelated factors. One manipulation, described as top-down attention, involved the request to respond either to features of the CSs or to features of the USs. A second manipulation, described as bottom-up attention, involved presentations of the CSs and USs either in the same modality (i.e., visual) or in different modalities (i.e., visual vs. auditory). Blask et al. found significant effects of both factors, such that conditions that facilitated attention to both stimuli produced stronger EC effects. However, whereas the effect of the topdown manipulation was mediated by recollective memory of the CS-US pairings, this was not the case for the effect of the bottom-up manipulation. In terms of the reviewed automaticity features, the top-down effect suggests that EC effects can be influenced by a goal to attend to and respond to the CS versus the US during the presentation of CS-US pairings. Moreover, the bottom-up manipulation is interesting, because to our knowledge it is the first demonstration of an attention effect that is difficult to attribute to the operation of a goal. Because both the bottom-up and the top-down manipulation were implemented during presentation of CS-US pairings, the results provide insights into the influence of these factors on the acquisition of conditioned evaluations.

Verwijmeren, Karremans, Stroebe, and Wigboldus, (2012, this issue) investigated the influence of a goal to drink (thirst) on the liking of beverages that were paired with USs that were either relevant (disgusted faces) or irrelevant (fearful faces) for the goal to drink. Their results showed that the disliking of the beverage paired with a goal-relevant US compared to the beverage paired with a goal-irrelevant US was stronger for thirsty 
than non-thirsty participants. These results provide insights into how goal states can moderate EC effects for goal-relevant stimuli. In these studies, the goal was active throughout the entire experiment. Therefore, it might have influenced EC effects through processes during the presentation of the stimulus pairings or during the measurement of CS evaluations (or both).

Gast, De Houwer, and De Schryver (2012, this issue) were particularly interested in the role of conscious knowledge of CS-US pairings during the measurement of CS evaluations. Although a positive relation between EC effects and knowledge of CS-US pairings has been shown in numerous studies, it is unclear whether this effect reflects the use of conscious knowledge during the expression of an evaluation or the role of conscious knowledge at earlier processing stages (e.g., during the CS-US pairings), which might decay over time. By measuring CS-US knowledge twice with a delay of several days, the authors demonstrated the relevance of contingency knowledge during the measurement of CS evaluations.

Unkelbach, Stahl, and Förderer (2012, this issue) investigated generalization in EC by testing whether CSs still produced significant EC effects when their visual appearance was modified to varying degrees. Although recognition accuracy for the modified stimuli remained at a very high level, EC effects emerged only for those CSs that were identical during learning and testing, but not for modified CSs. Moreover, when distinguishing between remembered and non-remembered pairs, this pattern emerged only for remembered CS-US pairs, suggesting that both memory and unchanged CS appearance were necessary preconditions for EC effects in this study. 
Perugini, Richetin, and Zogmaister (2012, this issue) investigated the potential impact of CS features on EC effects, more specifically whether EC effects are moderated by the pre-existing valence of the CSs. To address this question, the authors used a variant of the self-referencing task, a paradigm in which CSs and USs are not paired directly in a spatio-temporal manner, but indirectly through a shared key response. The authors showed that both neutral and valenced CSs can be conditioned with this task, and these effects were shown on both explicit and implicit measures.

Glaser and Walther (2012, this issue) investigated the influence of mixedvalenced USs. Toward this end, they used a picture-picture paradigm with portraits to investigate how evaluatively inconsistent features of the USs might differentially influence CS and US evaluations. Neutral CSs were paired with USs that varied in terms of their valence on a behavioral (drug use vs. no drug use) and a group-related dimension (membership in a likeable vs. a dislikeable group). The authors showed that, while the first US feature (drug use vs. no drug use) dominated the evaluation of the USs, the second US feature (membership in a likeable vs. dislikeable group) dominated the evaluation of the CSs they were paired with.

Finally, Zanon, De Houwer, and Gast (2012, this issue) investigated the moderating influence of contextual information on EC effects by presenting CS-US pairs as part of a slot machine game in which pairs of meaningless words were paired with winning and losing outcomes. In addition, participants were presented with context stimuli that implied the presence of a rule. Depending on the particular condition, this rule suggested an evaluation of the CS that was either congruent or incongruent with the valence of the US it was paired with. The authors showed that context cues that suggested 
a reversed CS valence attenuated EC effects on different implicit measures. The authors argue that these findings are consistent with both a dual-process account and a singleprocess propositional account of evaluative conditioning. Yet, they are inconsistent with a single-process associative account.

\section{Conclusion}

Over the past few years, research relying on functional definitions has produced significant insights into the nature of and the processes underlying EC. Although the question of how EC should be defined is still under debate, functional definitions greatly facilitated empirical tests of competing accounts by clearly distinguishing between EC as an effect and the mental processes and representations that underlie this effect. Increased methodological sophistication and the adoption of meta-conditional approaches have been instrumental in testing hypotheses about the mechanisms underlying EC, and these developments will likely shape EC research over the coming years. We assume that the distinction between processes that operate during acquisition and processes that operate during measurement will play a major role in this regard, with a particular focus on whether these processes operate in an automatic or non-automatic fashion. This Special Issue features a strong selection of articles that are characteristic for these trends, offering important new insights into topics such as the role of automaticity features in EC (e.g., conscious knowledge, goal-dependence, controllability), the relevance of stimulus characteristics (e.g., neutrality of CSs, mixed valence USs, CS feature similarity), and the influence of contextual information (e.g., contextual cues about CS-US relations). 


\section{References}

Baeyens, F., Eelen, P., \& Van den Bergh, O. (1990). Contingency awareness in evaluative conditioning: A case for unaware affective-evaluative learning. Cognition \& Emotion, 4, 3-18. doi:10.1080/02699939008406760

Balas, R., \& Gawronski, B. (2012). On the intentional control of conditioned evaluative responses. Learning and Motivation. [This issue].

Bar-Anan, Y., De Houwer, J., \& Nosek, B. A. (2010). Evaluative conditioning and conscious knowledge of contingencies: A correlational investigation with large samples. Quarterly Journal of Experimental Psychology, 63, 2313-2335. doi:10.1080/17470211003802442

Batchelder, W. H., \& Riefer, D. M. (1999). Theoretical and empirical review of multinomial process tree modeling. Psychonomic Bulletin \& Review, 6, 57-86. doi: 10.3758/BF03210812

Blask, K., Walther, E., Halbeisen, G., \& Weil, R. (2012). At the crossroads: Attention, contingency awareness, and evaluative conditioning. Learning and Motivation. [This issue].

Corneille, O., Yzerbyt, V. Y., Pleyers, G., \& Mussweiler, T. (2009). Beyond awareness and resources: Evaluative conditioning may be sensitive to processing goals. Journal of Experimental Social Psychology, 45, 279-282. doi:10.1016/j.jesp.2008.08.020

De Houwer, J. (2006). Using the implicit association test does not rule out an impact of conscious propositional knowledge on evaluative conditioning. Learning and Motivation, 37, 176-187. 
De Houwer, J. (2007). A conceptual and theoretical analysis of evaluative conditioning. The Spanish Journal of Psychology, 10, 230-241. Retrieved from http://www.ucm.es/info/psi/docs/journal/v10_n2_2007/art230.pdf

De Houwer, J. (2009). The propositional approach to associative learning as an alternative for association formation models. Learning \& Behavior, 37, 1-20. doi:10.3758/LB.37.1.1

De Houwer, J. (2011a). Why the cognitive approach in psychology would profit from a functional approach and vice versa. Perspectives on Psychological Science, 6, 202-209.doi: 10.1177/1745691611400238

De Houwer, J. (2011b). Evaluative conditioning: A review of functional knowledge and mental process theories. In T. R. Schachtman \& S. Reilly (Eds.), Associative learning and conditioning theory. Human and non-human applications (pp. 399416). Oxford, UK: Oxford University Press.

De Houwer, J., Baeyens, F., \& Field, A. P. (2005). Associative learning of likes and dislikes. Some current controversies and possible ways forward. Cognition and Emotion, 19, 161-174. doi:10.1080/02699930441000265

De Houwer, J., Thomas, S., \& Baeyens, F. (2001) Associative learning of likes and dislikes: A review of 25 years of research on human evaluative conditioning. Psychological Bulletin, 127, 853-869. doi: 10.1037//0033-2909.127.6.853

Dedonder, J., Corneille, O., Yzerbyt, V., \& Kuppens, T. (2010). Evaluative conditioning of high-novelty stimuli does not seem to be based on an automatic form of associative learning. Journal of Experimental Social Psychology, 46, 1118-1121. doi: 10.1016/j.jesp.2010.06.004 
Deutsch, R., Gawronski, B., \& Strack, F. (2006). At the boundaries of automaticity: Negation as reflective operation. Journal of Personality and Social Psychology, 91, 385-405. doi: 10.1037/0022-3514.91.3.385

Fazio, R. H., Sanbonmatsu, D. M., Powell, M. C., \& Kardes, F. R. (1986). On the automatic activation of attitudes. Journal of Personality and Social Psychology, 50, 229-238. doi: 10.1037//0022-3514.50.2.229

Ferguson, M. J., \& Bargh, J. A. (2004). Liking is for doing: The effects of goal pursuit on automatic evaluation. Journal of Personality and Social Psychology, 87, 557-572. doi: $10.1037 / 0022-3514.87 .5 .557$

Fiedler, K., \& Unkelbach, C. (2011). Evaluative conditioning depends on higher order encoding processes. Cognition and Emotion, 25, 639-656. doi: $10.1080 / 02699931.2010 .513497$

Field A. P. (2006). Is conditioning a useful framework for understanding the development and treatment of phobias? Clinical Psychology Review, 26, 857-875. doi:10.1016/j.cpr.2005.05.010

Field, A. P., \& Moore, A. C. (2005). Dissociating the effects of attention and contingency awareness on evaluative conditioning effects in the visual paradigm. Cognition \& Emotion, 19, 217-243. doi: 10.1080/02699930441000292

Förderer, S., \& Unkelbach, C. (2012). Hating the cute kitten or loving the aggressive pitbull: EC effects depend on CS-US relations. Cognition and Emotion, 26, 534-540. doi: 10.1080/02699931.2011.588687 
Fulcher, E. P., \& Hammerl, M. (2001). When all is revealed: A dissociation between evaluative learning and contingency awareness. Consciousness and Cognition, 10, 524-549. doi: 10.1006/ccog.2001.0525

Gast, A., De Houwer, J., \& De Schryver, M. (2012). Evaluative conditioning can be modulated by memory of the CS-US pairings at the time of testing. Learning and Motivation. [This issue].

Gast, A., \& Rothermund, K. (2011a). I like it because I said that I like it. Evaluative conditioning effects can be based on stimulus-response learning. Journal of Experimental Psychology: Animal Behavior Processes, 37, 466476.doi:10.1037/a0023077

Gast, A., \& Rothermund, K. (2011b). What you see is what will change: Evaluative conditioning effects depend on a focus on valence. Cognition \& Emotion, 25, 89110. doi:10.1080/02699931003696380

Gawronski, B., \& Bodenhausen, G. V. (2006). Associative and propositional processes in evaluation: An integrative review of implicit and explicit attitude change. Psychological Bulletin, 132, 692-731. doi:10.1037/0033-2909.132.5.692

Gawronski, B., \& Bodenhausen, G. V. (2011). The associative-propositional evaluation model: Theory, evidence, and open questions. Advances in Experimental Social Psychology, 44, 59-127. doi:10.1016/B978-0-12-385522-0.00002-0

Gawronski, B., \& Bodenhausen, G. V. (in press). The associative-propositional evaluation model: Operating principles and operating conditions of evaluation. In J. W. Sherman, B. Gawronski, \& Y. Trope (Eds.), Dual-process theories of the social mind. New York: Guilford Press. 
Gawronski, B., \& De Houwer, J. (in press). Implicit measures in social and personality psychology. In H. T. Reis, \& C. M. Judd (Eds.), Handbook of research methods in social and personality psychology (2nd edition). New York: Cambridge University Press.

Gawronski, B., Deutsch, R., Mbirkou, S., Seibt, B., \& Strack, F. (2008). When “just say no" is not enough: Affirmation versus negation training and the reduction of automatic stereotype activation. Journal of Experimental Social Psychology, 44, 370-377. doi: 10.1016/j.jesp.2006.12.004

Gawronski, B., \& LeBel, E. P. (2008). Understanding patterns of attitude change: When implicit measures show change, but explicit measures do not. Journal of Experimental Social Psychology, 44, 1355-1361. doi: 10.1016/j.jesp.2008.04.005

Gawronski, B., \& Walther, E. (2012). What do memory data tell us about the role of contingency awareness in evaluative conditioning? Journal of Experimental Social Psychology, 48, 617-623. doi:10.1016/j.jesp.2012.01.002

Glaser, T., \& Walther, E. (2012). One but not the same: Evaluative conditioning with mixed-valence USs. Learning and Motivation. [This issue].

Hayes, S. C., Barnes-Holmes, D., \& Roche, B. (2001). Relational frame theory: A postSkinnerian account of human language and cognition. New York: Plenum.

Hofmann, W., De Houwer, J., Perugini, M., Baeyens, F., \& Crombez, G. (2010). Evaluative conditioning in humans: A meta-analysis. Psychological Bulletin, 136, 390-421. doi: 10.1037/a0018916

Hütter, M., Sweldens, S., Stahl., C., Unkelbach, C., \& Klauer, K. C. (in press). Dissociating contingency awareness and conditioned attitudes: Evidence of 
contingency-unaware evaluative conditioning. Journal of Experimental Psychology: General.

Johnson, B. T., Maio, G. R., \& Smith-McLallen, A. (2005). Communication and attitude change: Causes, processes, and effects. In D. Albarracín, B. T. Johnson, \& M. P. Zanna (Eds.), Handbook of attitudes and attitude change (pp. 617-669). Mahwah, NJ: Erlbaum.

Jones, C. R., Fazio, R. H., \& Olson, M. A. (2009). Implicit misattribution as a mechanism underlying evaluative conditioning. Journal of Personality and Social Psychology, 96, 933-948. doi:10.1037/a0014747

Jones, C. R., Olson, M. A., \& Fazio, R. H. (2010). Evaluative conditioning: The "how" question. Advances in Experimental Social Psychology, 43, 205-255. doi: $10.1016 / \mathrm{S} 0065-2601(10) 43005-1$

Kattner, F. (2012). Revisiting the relation between contingency awareness and attention: Evaluative conditioning relies on a contingency focus. Cognition and Emotion, 26, 166-175. doi: 10.1080/02699931.2011.565036

Levey, A. B., \& Martin, I. (1975). Classical conditioning of human 'evaluative' responses. Behaviour Research and Therapy, 4, 205-207. doi: 10.1016/00057967(75)90026-1

Mineka, S., \& Zinbarg, R. (2006). A contemporary learning theory perspective on the etiology of anxiety disorders: It's not what you thought it was. American Psychologist, 61, 10-26. doi: 10.1037/0003-066X.61.1.10 
Mitchell, C. J., De Houwer, J., \& Lovibond, P. F. (2009). The propositional nature of human associative learning. Behavioral and Brain Sciences, 32, 183-198. doi:10.1017/S0140525X09000855

Moors, A., \& De Houwer, J. (2006). Automaticity: A theoretical and conceptual analysis. Psychological Bulletin, 132, 297-326. doi:10.1037/0033-2909.132.2.297

Moors, A., Spruyt, A., \& De Houwer, J. (2010). In search of a measure that qualifies as implicit. Recommendations based on a decompositional view of automaticity. In B. Gawronski, \& B. K. Payne (Eds.), Handbook of implicit social cognition: Measurement, theory, and applications (pp. 19-37). New York, NY: Guilford Press.

Payne, B. K., Cheng, S. M., Govorun, O., \& Stewart, B. D. (2005). An inkblot for attitudes: Affect misattribution as implicit measurement. Journal of Personality and Social Psychology, 89, 277-293. doi: 10.1037/0022-3514.89.3.277

Perugini, M., Richetin, J., \& Zogmaister, C. (2012). The formation of implicit and explicit attitudes for neutral and valenced stimuli using the self. Learning and Motivation. [This issue].

Pleyers, G., Corneille, O., Luminet, O., \& Yzerbyt, V. (2007). Aware and (dis)liking: Item-based analyses reveal that valence acquisition via evaluative conditioning emerges only when there is contingency awareness. Journal of Experimental Psychology: Learning, Memory, and Cognition, 33, 130-144. doi:10.1037/02787393.33.1.130 
Pleyers, G., Corneille, O., Yzerbyt, V., \& Luminet, O. (2009). Evaluative conditioning may incur attentional costs. Journal of Experimental Psychology: Animal Behavior Processes, 35, 279-285. doi:10.1037/a0013429

Popper, K. R. (1934). Logic of scientific discovery. New York: Basic Books.

Purkis, H. M., \& Lipp, O. V. (2001). Does affective learning exist in the absence of contingency awareness? Learning and Motivation, 32, 84-99. doi:10.1006/lmot.2000.1066

Seibt, B., Häfner, M., \& Deutsch, R. (2007). Prepared to eat: How immediate affective and motivational responses to food cues are influenced by food deprivation. European Journal of Social Psychology, 37, 359-379. doi: 10.1002/ejsp.365

Shanks, D. R., \& St. John, M. F. (1994). Characteristics of dissociable human learning systems. Behavioural and Brain Sciences, 17, 367-447. doi:10.1017/S0140525X00035032

Stahl, C., Unkelbach, C., \& Corneille, O. (2009). On the respective contributions of awareness of unconditioned stimulus valence and unconditioned stimulus identity in attitude formation through evaluative conditioning. Journal of Personality and Social Psychology, 97, 404-420. doi:10.1037/a0016196

Sweldens, S., Van Osselaer, S., \& Janiszewski, C. (2010). Evaluative conditioning procedures and the resilience of conditioned brand attitudes. Journal of Consumer Research, 37, 473-489. doi: 10.1086/653656

Unkelbach, C., Stahl, C., \& Förderer, S. (2012). Changing CS features alters evaluative responses in evaluative conditioning. Learning and Motivation. [This issue]. 
Verwijmeren, T., Karremans, J. C., Stroebe, W., \& Wigboldus, D. H. J. (2012). Goal relevance moderates evaluative conditioning effects. Learning and Motivation. [This issue].

Walther, E. (2002). Guilty by mere association: Evaluative conditioning and the spreading attitude effect. Journal of Personality and Social Psychology, 82, 919934. doi:10.1037/0022-3514.82.6.919

Walther, E., Gawronski, B., Blank, H., \& Langer, T. (2009). Changing likes and dislikes through the backdoor: The US revaluation effect. Cognition and Emotion, 23, 889-917. doi: 10.1080/02699930802212423

Zanon, R., De Houwer, J., \& Gast, A. (2012). Context effects in evaluative conditioning of implicit evaluations. Learning and Motivation. [This issue]. 


\footnotetext{
${ }^{1}$ An important difference between the definition by De Houwer (2007) and most other definitions is that the former does not refer to the initial valence of the stimuli that are paired. Requiring that one of the stimuli has to be valenced takes into account what is seen as the prototypical case of EC, but it also imposes a priori restrictions on its properties. For example, if EC is limited to changes in liking of neutral stimuli that are paired with valenced stimuli, phenomena such as sensory pre-conditioning (i.e., a change in liking of CS1 after experiencing CS1-CS2 trials that are followed by CS2-US trials; e.g., Hammerl \& Grabitz, 1996; Walther, 2002) would not qualify as instances of EC. A further difference between the definition by De Houwer and some other definitions is that it does not refer to the direction in which liking changes. Hence, even instances in which the change in liking of a stimulus is not in the direction of the existing valence of the other stimuli qualify as instances of EC (i.e., reverse EC effects).

${ }^{2} \mathrm{~A}$ similar argument is sometimes made against the use of stimulus pairings that suggest the presence of propositional processes. For example, the effects of pairing an image of a person (CS) with a word denoting a personality trait (US) might be rejected as an instance of EC, because it suggests a specific relation between the person and the personality trait (i.e., that person possesses the trait). Similar to our arguments about instruction effects, we argue that the absence of propositional processes is not a suitable criterion for defining EC effects.
} 